\title{
Targeting the S1P/S1PR1 axis mitigates cancer-induced bone pain and neuroinflammation
}

Authors: Shaness A. Grenald ${ }^{1}$, Timothy M. Doyle ${ }^{2}$, Hong Zhang ${ }^{1}$, Lauren M. Slosky1 ${ }^{1}$ Zhoumou Chen², Tally M. Largent-Milnes ${ }^{1}$, Sarah Spiegel ${ }^{3}$, Todd W. Vanderah ${ }^{1}$ and Daniela Salvemini ${ }^{2, \$}$

1Department of Pharmacology, University of Arizona, 1501 N Campbell Ave, Tucson, AZ 85724, USA 2Department of Pharmacology and Physiology, Saint Louis University School of Medicine, 1402 South Grand Blvd, St. Louis, MO 63104, USA

3Department of Biochemistry and Molecular Biology, Virginia Commonwealth University, 1101 E. Marshall Street, Richmond, VA 23298, USA

Number of Pages: 17

Number of Figures: 6

§Corresponding author: E-mail: salvemd@ @lu.edu, Phone: 1-314-977-6430, Fax: 1-314-977-6441, Address: 1402 South Grand Blvd, St. Louis, MO 63104, USA

Short title: S1PR1 antagonists in cancer-induced bone pain

\begin{abstract}
Metastatic bone pain is the single most common form of cancer pain, and persists as a result of peripheral and central inflammatory, as well as, neuropathic mechanisms. Here, we provide the first characterization of sphingolipid metabolism alterations in the spinal cord occurring during cancer-induced bone pain (CIBP). Following femoral arthrotomy and syngenic tumor implantation in mice, ceramides decreased with corresponding increases in sphingosine and the bioactive sphingolipid metabolite, sphingosine 1-phosphate (S1P). Intriguingly, de novo sphingolipid biosynthesis was increased as shown by the elevations of dihydroceramides and dihydro-S1P. We next identified the S1P receptor subtype 1 (S1PR1) as a novel target for therapeutic intervention. Intrathecal or systemic administration of the competitive and functional S1PR1 antagonists, TASP0277308 and FTY720/Fingolimod, respectively, attenuated cancer-induced spontaneous flinching and guarding. Inhibiting CIBP by systemic delivery of FTY720 did not result in antinociceptive tolerance over 7 days. FTY720 administration enhanced IL-10 in the lumbar ipsilateral spinal cord of CIBP animals, and intrathecal injection of an IL-10 neutralizing antibody mitigated the ability of systemic FTY720 to reverse CIBP. FTY720 treatment was not associated with alterations in bone metabolism in vivo. Studies here identify a novel mechanism to inhibit bone cancer pain by blocking the actions of the bioactive metabolites S1P and dihydro-S1P in lumbar spinal cord induced by bone cancer and support potential fast-track clinical application of the FDA-approved drug, FTY720, as a therapeutic avenue for CIBP.
\end{abstract}

Key words: S1PR1 antagonists, FTY720, TASP0277308, IL-10 


\section{Introduction}

Cancer pain encompasses all pain states due to tumor occupation of soft tissue or bone and peripheral neuropathy resultant from chemotherapeutic treatment [21]. Bone pain (cancer-induced bone pain, CIBP) is reported by $30-50 \%$ of all cancer patients and by $75-90 \%$ of late-stage patients [50]. CIBP is driven by a combination of peripheral (tumor-associated) skeletal, inflammatory and neuropathic mechanisms [36]. Innovations in the treatment of bone cancer pain have primarily focused on the development of adjunct therapeutics addressing secondary bone loss and vulnerability to painful skeletal-related events. However, no therapeutic strategies to date target the neuropathic mechanisms of CIBP. In a clinical study, 24\% of patients with metastatic bone pain exhibited neuropathic features [45]. Furthermore, the persistence of neuropathic characteristics following remission is hypothesized to underlie continued pain in recovering patients [10]. Thus, the need for efficacious therapeutic interventions rather than palliative care is crucial as the proportion of patients recovering from metastatic cancer increases; better understanding of the neuropathic features and therapeutic targets of CIBP are critical to the development of such strategies.

Ceramide is a pro-inflammatory, pro-apoptotic sphingolipid whose metabolism has recently been associated with pain $[51 ; 57]$. Ceramide is formed through de novo synthesis from condensation of palmitate and serine to form dihydrosphingosine, which is acylated to dihydroceramides and then desaturated to ceramides. Catabolism of sphingomyelin and glycosylated ceramides in the lysosome also generates ceramide through the salvage pathway [31]. Ceramides are degraded to sphingosine that can be phosphorylated by sphingosine kinase to sphingosine-1-phosphate (S1P), a potent inflammatory sphingolipid metabolite [54] that has a causative role in a variety of persistent pain states by binding to the S1P receptor 1 (S1PR1) in the nervous system [51]. In addition to the role of the ceramide-to-S1P pathway in peripheral inflammation and pain [16; 18], elevated ceramide and S1P levels in the spinal cord have been suggested to play a critical role in neuropathic pain $[26 ; 47]$. For example, elevations in spinal S1P mediate the induction and maintenance of paclitaxel-induced peripheral neuropathic pain that can be prevented or attenuated by the administration of S1P receptor subtype 1 antagonists [26]. However, there is little known about these sphingolipid metabolites in CIBP.

Here, we propose an alteration in spinal sphingolipid content may underlie the maintenance of CIBP in a syngeneic 66.1-BALB/c murine breast cancer metastasis model. In the lumbar region ipsilateral to the tumorbearing limb, we observed changes in the sphingolipid salvage pathway that lead to dihydro-S1P synthesis, another ligand of S1PR1. Moreover, increased degradation of ceramides leads to increases in S1P and accordingly, blockade of spinal S1PR1 signaling profoundly attenuated spontaneous cancer-related pain behaviors. We demonstrate for the first time the bioactivity of spinal sphingolipid metabolites S1P and dihyroS1P and the ensuing neuroinflammation are critical components of CIBP. These findings identify S1PR1 as a potential therapeutic target alone or as an adjunct therapy to address the neuropathic qualities of CIBP. 


\section{Materials and Methods}

\subsection{Experimental Animals}

Female BALB/c mice (18-20g; Harlan, IN, USA) were housed in a climate-controlled specific pathogen-free room (12-h light/dark cycle) with ad libitum access to food and water. Estrous cycles were not controlled because the study (14 days) was longer than the 4-5 days estrous cycle and female mice were housed separately from male mice to induce assumed anestrus [1]. Studies were conducted by a blinded observer between the hours of 08:00-12:00 in accordance with the International Association for the Study of Pain, the National Institutes of Health and with the approval of the Saint Louis University and University of Arizona Institutional Animal Care and Use Committees.

\subsection{Cell Culture}

Murine 66.1 cells were grown at $37^{\circ} \mathrm{C}$ under $5 \% \mathrm{CO}_{2}$ in Eagle's minimum essential medium (Mediatech, Manassas, VA), containing $10 \%$ fetal bovine serum, $100 \mathrm{IU} / \mathrm{ml}$ penicillin and $100 \mu \mathrm{g} / \mathrm{mL}$ streptomycin, as previously described [36]. All in vivo and in vitro assays used cells passaged 10-20 times.

\subsection{Femoral arthrotomy}

Mouse femoral arthrotomy was performed as previously described by our laboratory [35]. Briefly, animals were anesthetized $(80 \mathrm{mg} / \mathrm{kg}$ ketamine, $12 \mathrm{mg} / \mathrm{kg}$ xylazine in $10 \mathrm{ml} / \mathrm{kg}$, i.p.) and the condyles of the right distal femur were exposed. The femur was arthrotomized and a placement needle was inserted to verify access to the intramedullary space via radiographic imaging (Faxitron, Lincolnshire, IL, USA). A suspension of 66.1 mammary adenocarcinoma cells $\left(4 \times 10^{5}\right)$ in growth medium or growth medium alone was injected into the intramedullary space of the femur and the injection site was sealed with dental amalgam. Animals were allowed a 7-day period for surgical recovery and tumor manifestation. All treatments were initiated on or after postsurgery day 7. For intrathecal drug administration, animals were lightly anesthetized with $3 \%$ isoflurane/ $\mathrm{O}_{2}$ inhalation (maintained on $2 \%$ isoflurane $/ \mathrm{O}_{2}$ ), the skin over the lumbar region of the spine was shaved, disinfected, and a percutaneous injection into the lumbar intrathecal space was performed. For subcutaneous infusion studies, animals were anesthetized as described above and a small $(<1 \mathrm{~cm})$ incision was made between the shoulder blades where a primed subcutaneous osmotic minipump (Alzet \#1007D; $0.5 \mu 1 /$ hour for 7 days) containing drug or vehicle control was inserted. The incision was closed with surgical adhesive and the animals were monitored post-operatively. 


\subsection{Test Compounds}

TASP0277308 was synthesized as previously reported [19]. Fingolimod (FTY720) was purchased from Cayman Chemical (Ann Arbor, MI, USA). Sheep anti-rat IL-10 IgG antibody was a generous gift from Dr. Linda Watkins at the University of Colorado Boulder. The rat IL-10 neutralizing antibodies were raised in sheep at the National Institute of Biological Standards and Control (South Mimms, Hertfordshire, UK) and purified by Avigen (Alameda, CA, USA) and have been shown to specifically block IL-10 signaling when intrathecally administered [34; 53]. Control sheep serum IgG was obtained from Sigma Aldrich (St. Louis, MO, USA).

\subsection{Behavioral Testing}

Pain behaviors were measured in mice prior to surgery (day 0, baseline, BL) and evaluated again on postsurgery day 10 for acute treatment studies or on days 7, 10 and 14 for continuous treatment studies. To evaluate spontaneous pain behaviors, mice were acclimated for 30 minutes in individual chambers with a wire mesh floor. Flinching and guarding behaviors were then observed over 2 minute periods. Flinching was characterized by rapid flexion and lifting of the right hind paw when not associated with locomotion. Guarding was characterized by the retraction of the right hind limb under the torso. CIBP is defined as a significant difference in the number of flinches or seconds guarding (typically $\geq 5$ flinches and $\geq 5$ seconds guarding over a 2-minute period) when compared to behavior prior to femoral arthrotomy on day 0. When behavior is expressed as \%Reversal of CIBP, the data for each animal was normalized against its baseline (day 0) and its peak pain (day 7) according to the following equation: \%Reversal of CIBP $=$ (behavior at $t$ - behavior at day 7) / (behavior at day 0 - behavior at day 7) x 100, where $t$ is the time point measured.

\subsection{Sphingolipid analysis by mass spectrometry}

Portions of mouse spinal cords from lumbar region ipsilateral to femoral injection site of 66.1 cells were harvested and placed into $13 \times 100 \mathrm{~mm}$ borosilicate tubes with a Teflon-lined cap (VWR, West Chester, PA). Lipids were extracted by adding $2 \mathrm{ml}$ of $\mathrm{CH}_{3} \mathrm{OH}$ and $1 \mathrm{ml}$ of $\mathrm{CHCl}_{3}$ together with the internal standard cocktail from Avanti Polar Lipids (Alabaster, AL) which was added to samples in $20 \mu$ ethanol:methanol:water (7:2:1) as a cocktail of 500 pmol each. Standards for sphingoid bases and sphingoid base 1-phosphates were 17-carbon chain length analogs: C17-sphingosine; C17-dihydrosphingosine; C17-sphingosine-1-phosphate (S1P); and C17-dihydrosphingosine-1-phosphate (dihydro-S1P). Standards for N-acyl sphingolipids were C12-fatty acid analogs: C12-Cer, N-(dodecanoyl)-sphing-4-enine (d18:1/C12:0); C12-Cer 1-phosphate, N-(dodecanoyl)sphing-4-enine-1-phosphate (d18:1/C12:0-Cer1P); C12-sphingomyelin, N-(dodecanoyl)-sphing-4-enine-1phosphocholine (d18:1/C12:0-SM); and C12-glucosylceramide, N-(dodecanoyl)-1- $\beta$-glucosyl-sphing-4-enine. Samples were dispersed by sonication and incubated at $48^{\circ} \mathrm{C}$ overnight. After cooling, $150 \mu \mathrm{l}$ of $1 \mathrm{M} \mathrm{KOH}$ in $\mathrm{CH}_{3} \mathrm{OH}$ was added to cleave glycerophospholipids. Extracts were neutralized with $12 \mu \mathrm{l}$ of glacial acetic acid, 
centrifuged, supernatants transferred to new tubes and evaporated to dryness with a speed vac. Residues were reconstituted in $0.5 \mathrm{ml}$ of starting mobile phase solvent for liquid chromatography-electrospray ionizationtandem mass spectrometry (LC-ESI-MS/MS), sonicated, centrifuged, and clear supernatants transferred to autoinjector vials for analyses. Sphingolipids were separated by reverse-phase HPLC coupled to an AB Sciex 5500 quadrupole/linear ion trap (QTrap; SCIEX Framingham, MA) operating in triple quadrupole mode, as described previously [9].

\subsection{Cytokines microbead assay}

The levels of cytokines within the dorsal lumbar spinal cord were assessed using a commercially available magnetic multiplex cytokine kit (Bio-Rad Laboratories). Samples were processed as previously described [25], according to manufacturer's protocol.

\subsection{Bone Loss Scoring}

Digital radiographs of the lower extremities were taken following behavioral testing on days 0, 7 and 14 (MX20 DC12, Faxitron XRay, Lincolnshire, IL, USA). Bone loss was rated by an observer blinded to treatment group according to a modified 5 point scale: $0=$ normal; 1 = small radiolucent lesions, indicative of bone destruction (1-3 lesions); 2 = increased number of lesions (3-6 lesions); 3 = full-thickness unicortical fracture; $4=$ fullthickness bicortical fracture [4]. Animals receiving a score of 4 were humanely euthanized and excluded from the study.

\subsection{Serum Biochemical Assay}

Immediately following behavioral testing on day 14, mice were anesthetized $(80 \mathrm{mg} / \mathrm{kg}$ ketamine and 12 $\mathrm{mg} / \mathrm{kg}$ xylazine in $10 \mathrm{ml} / \mathrm{kg}$, i.p.) and whole blood was collected by transcardial puncture. Blood was allowed to coagulate at room temperature for 1 hour before isolating serum by centrifugation. Serum was stored at $-80^{\circ} \mathrm{C}$ until use. Serum C-telopeptide fragment of collagen type I (CTX) was quantified using an ELISA according to manufacturer specifications (Immunodiagnostic Systems, Gaithersburg, MD, USA) with a detection limit of 2 $\mathrm{ng} / \mathrm{ml}$ and $50 \mathrm{ng} / \mathrm{ml}$.

\subsection{Statistical Analysis of Data}

Data are expressed as mean \pm SEM for $n$ animals. The $\mathrm{ED}_{50}$ values for the reversal of flinching and guarding behavior by test compounds were determined by a three-parameter, non-linear regression analysis of normalized data. Time-dependent behavior was analyzed by a two-tailed, two-way ANOVA with Bonferroni post hoc comparisons. Pairwise comparisons were made using a Welch's corrected, unpaired, one-tailed Student's t-test. The false discovery rate (FDR) for the multiple pairwise comparisons of the lipidomic data was controlled by Benjamini-Hochberg $(\mathrm{B}-\mathrm{H})$ procedure $(\mathrm{q}<0.05)$. Significant differences were defined as $\mathrm{p}<0.05$ for all data 
except lipidomic data where significance was defined at $\mathrm{p}<0.032$. All statistical analyses were performed using GraphPad Prism 6.0 (Graph Pad Inc., San Diego, CA) or IBM SPSS Statistics for Windows, Version 21.0. IBM, Armonk, NY).

\section{Results}

\subsection{CIBP enhances spinal cord levels of dihydro-S1P and S1P}

To examine the relevance of central sphingolipids in metastatic cancer pain, we profiled the sphingolipidomic changes in the lumbar ipsilateral spinal cord of female mice by LC-ESI-MS/MS 14 days after femoral arthrotomy and inoculation of spontaneously-occurring murine breast cancer 66.1 cells, a commonly used model of CIBP [35]. There were significant reductions in the levels of almost all ceramide species (Fig. 1A) and monohexosylceramides (Fig. 1B), yet no major changes in levels of sphingomyelin were observed (Fig. 1C). These data suggest that ceramide degradation by ceramidase(s) that cleave all ceramide acyl chain species is increased in the lumbar spinal cord of bone tumor bearing mice. This was accompanied by increased levels of sphingosine, a product of ceramidase activity (Fig. 1D), and a corresponding increase in S1P (Fig. 1E), catalyzed by phosphorylation of sphingosine by sphingosine kinase. In contrast, there were significant increases of dihydroceramides, an intermediate in de novo sphingolipid biosynthesis (Fig. 2A), and monohexosyldihydroceramides (Fig. 2B) but not dihydrosphingomyelins (Fig. 2C). Intriguingly, while dihydrosphingosine remained unchanged (Fig. 2D), significant increases of dihydro-S1P (Fig. 2E) in the spinal cord were observed, further indicating sphingosine kinase activation in the spinal cord during CIBP.

\subsection{Intrathecal and systemic administration of $S 1 P R 1$ antagonists reverses CIBP pain behaviors}

Because both S1P and dihydro-S1P are ligands of S1PR1 and our laboratory has previously demonstrated that S1PR1 mediates the neuropathic and pro-nociceptive qualities of S1P in the spinal cord [26; 51], we next evaluated whether increases in S1P and dihydro-S1P are functionally relevant in CIBP by pharmacologically targeting the S1PR1. Following the establishment of CIBP (day 11), animals were administered an intrathecal injection of vehicle, S1PR1 antagonist TASP0277308 [3] or functional antagonist FTY720 [3]. Administration of TASP0277308 or FTY720, but not their vehicle, rapidly ( $\leq 30 \mathrm{~min}$ ) reversed flinching and guarding behavior with an effect that peaked within $30 \mathrm{~min}$ and resolved by $3 \mathrm{~h}$ (Figs. 3A, B). Whereas these results suggest a spinal site of action of the S1PR1 antagonists, we cannot of course exclude potential contributing effects of S1PR1 antagonists on S1PR1 expressed on DRG neurons and immune cells as some drugs are known to access the DRG site post i.th. injection [51].

To evaluate the clinical applicability of a S1PR1-targeted strategy, FTY720 was administered systemically to CIBP animals. FTY720 rapidly ( $\leq 30 \mathrm{~min})$ and dose-dependently reversed flinching and guarding behavior with an effect that peaked within $1.5 \mathrm{~h}$ and resolved by $6 \mathrm{~h}$ (Figs. 3C, D). Animals displayed no qualitative signs of sedation or motor impairment. 


\subsection{Continuous reversal of CIBP by S1PR1 antagonists requires spinal IL-10 signaling}

$\mathrm{CIBP}$ is a progressive pain state; therefore therapies are highly vulnerable to the development of tolerance and loss of their efficacy to treat CIBP. Indeed, the clinical failure of current therapies is most often due to antinociceptive tolerance and disease-related pain progression [33; 48]. Therefore, on days 7-14, we administered either a single daily injection of FTY720 (1 mg/kg/d, i.p., Figs. 4A, B) or continuous subcutaneous infusion of FTY720 (1 mg/kg/d, s.c. osmotic minipump, Figs. 5A, B) to CIBP animals. In both therapeutic paradigms, FTY720, but not its vehicle, significantly attenuated flinching and guarding behaviors when measured on days 7 and 14. There was no significant decrease in the antinociceptive efficacy of FTY720 observed over a 7-day period.

\subsection{Involvement of IL-10 in suppression of CIBP by FTY720}

To determine the mechanisms involved in S1PR1-mediated continuous antinociception in CIBP, we investigated the previously reported anti-neuroinflammatory effects of FTY720 [7] in our model of CIBP. Using a microbead cytokine screen (Bio-Rad, Hercules, CA, USA), the treatment-associated alterations in TNF, IL-1 $\beta$, IL-6, IL-10, and MCP-1 levels in the ipsilateral lumbar spinal cord on day 14 of CIBP were measured. Preliminary overview of the changes revealed that beneficial effects of FTY720 given subcutaneously via an implanted minipump were associated with attenuation of spinal expression of pro-inflammatory IL-1 $\beta$ and promoted spinal expression of anti-inflammatory IL-10 relative to untreated tumor-bearing controls (not shown). To evaluate the functional relevance of spinal IL-10 in FTY720-mediated relief of CIBP, we coadministered an intrathecal IL-10 neutralizing antibody or IgG control on days 10 and 14 during continuous subcutaneous infusion of FTY720. In previous rodent pain studies by Sloane et al., an intrathecal dose of this IL-10 neutralizing antibody blocked the ability of recombinant IL-10 overexpression in the spinal cord to provide short and long-term reversal of mechano-allodynia within hours of its administration [53]. Here, intrathecal administration of IL-10 neutralizing antibody, but not IgG control, rapidly ( $\leq 60 \mathrm{~min}$ ), completely, and reversibly abrogated FTY720 antinociception on both days 10 and 14 (Figs. 5A, B) indicating that the S1PR1 blockade elicited continuous IL-10 signaling as a mechanism of pain relief in CIBP.

\subsection{Reversal of CIBP by FTY720 is unrelated to changes in bone growth}

Multiple peripheral and central generators of nociception contribute to the state of CIBP [17]. To control for bone and tumor-related effects of FTY720, radiographs were taken on days 0, 7 and 14. Neither FTY720 (1 $\mathrm{mg} / \mathrm{kg} / \mathrm{d}$, i.p.) nor its vehicle (5\% DMSO control) affected the number of observed translucent (osteolytic) lesions in tumor-bearing animals; similarly no effects were observed in the sham control group (Figs. 6A,B). In serum harvested from these same animals on day 14, tumor burden was associated with an elevation in collagen-type I fragment (CTX) as compared to sham control (Fig. 6C), but FTY720 treatment did not alter 
circulating CTX in sham or tumor-bearing animals (Fig. 6C). These data suggest that repeated dosing of FTY720 does not alter bone metabolism in CIBP and therefore did not produce anti-nociception by reducing the frequency of skeletal-related events.

\section{Discussion}

Despite its prevalence, metastatic bone pain is poorly managed in patients using combinations of opioids and adjunct therapeutics [10]. The prevalence of bone pain is largely due to the propensity of epithelial-origin cancers to colonize the heavily vascularized areas of the skeleton, such as the red marrow of the long bones, sternum, pelvis, ribs and vertebrae [6]. Using a mammary adenocarcinoma (66.1 breast cancer cells) from a $\mathrm{BALB} / \mathrm{c}$ mouse and placing the cells into the intramedullary space of the femur, we create a syngenic (immunocompetent) murine model of breast cancer-induced bone metastasis [35] with the dysregulation of bone resorption and deposition that yields osteoclastic and osteoblastic lesions [43] and spontaneous pain similar to human metastatic bone cancers [43]. The use of this syngenic model and a murine species prone to inflammation [41] better represents the pro-inflammatory state seen in human metastatic cancers [42]. Unfortunately, cancer pain is ineffectually managed with non-steroidal anti-inflammatory drugs and opioids in $>30 \%$ of patients [55]. WHO guidelines for cancer pain management have remained unchanged since 1996 [58], which underscores the need for novel therapeutic development. Characterizations of CIBP in vivo have demonstrated the formation of peripheral neuropathic features at the site of bone-tumor burden, such as neuroma formation and the disruption of periosteal innervation [28; 38], as well as central neuropathic pathology in the dorsal horn [23; 60] and spinal glia [62]. In a clinical study, 24\% of patients with metastatic bone pain exhibited neuropathic features [45]. Furthermore, the persistence of neuropathic characteristics following remission is hypothesized to underlie continued pain in recovering patients [10]. Results presented in this manuscript unravel a previously unrecognized role for dysregulated sphingolipid metabolism and the S1PR1 axis in the central nervous system in neuropathic characteristics of CIBP using an established model of breast-origin CIBP [35].

For the first time, we demonstrate that painful peripheral bone tumor burden elicited alterations in spinal cord sphingolipid metabolism. These changes may represent a novel central neuropathic characteristic of CIBP. When compared to sham control animals, intra-femur cancer animals displayed consistent decreases in monohexosylceramides and ceramides in tandem with increases in pro-nociceptive S1P in the lumbar spinal cord ipsilateral to the neuro-innervation of femur tumor burden. The decrease of ceramide and its glycosylated metabolites is consistent with enhanced lysosomal degradation and the salvage pathway [31]. On the other hand, the increase in dihydroceramides implicates increased de novo sphingolipid biosynthesis in a manner that favors the formation of dihydro-S1P. It is possible that the ipsilateral input from the femur-innoculated cancer cells may alter sphingolipid metabolism in other spinal cord segments (i.e., thoracic or cervical cord); however, our studies focused on the ipsilateral lumbar cord due to the rapid behavioral response of FTY720 after 
intrathecal administration ( $\leq 30 \mathrm{~min})$. Altered spinal sphingolipid metabolism occurs in neuropathic rodents [26; $29 ; 47 ; 51 ; 57]$ and is implicated in neuronal cell death following both nerve injury [14] and ischemiareperfusion injury [15]. Moreover, S1P levels in the tumor microenvironment are greatly increased by sphingolipid metabolism in tumor cells, erythrocytes and platelets that in turn promote tumor migration, proliferation and angiogenesis [44; 49]. It is important to note that while 66.1 cells implanted and sealed into the bone do not metastasize within the 14-day test period in the absence of fracture [32] and are not likely to contribute directly to increased S1P in the spinal cord, local increases in S1P in the tumor-bearing bone could sensitize peripheral afferents $[11 ; 30 ; 46]$ and promote the development of CIBP and spinal S1P production by provoking adaptive glutamatergic and neuroinflammatory changes in the spinal cord [51]. S1P has emerged as a key regulatory molecule in breast cancer [22; 37; 49] and thus, reduction of its levels by FTY720 and/or altering S1P signaling may reduce breast cancer growth and bone metastasis and may partially be responsible for the decrease in CIBP, supporting its clinical use in metastatic cancers.

The functional relevance of increases in spinal cord levels of S1P and dihydro-S1P was validated using the S1PR1 antagonist TASP0277308 and the functional antagonist FTY720. FTY720 is an orally bioavailable highly CNS-permeant [5;7] drug that is currently approved for the treatment of relapsing-remitting multiple sclerosis [24]. Importantly, FTY720 requires phosphorylative bioactivation by sphingosine kinase 2 [8; 52]. Binding of FTY720-P to S1PR1 results in its rapid ubiquitination, proteasomal degradation, and irreversible down-regulation [20; 56] and accordingly, explains the functional antagonistic actions of FTY720 [3]. In order to control for the bioactivation requirement and functional antagonist nature of FTY720, TASP0277308 was selected as an S1PR1-specific competitive antagonist that does not require bioactivation. Indeed, FTY720 and TASP0277308 demonstrated near-identical reversal of CIBP pain behaviors, validating the importance of S1PR1 signaling in CIBP.

Critically, our data demonstrates that FTY720 provides continuous relief from CIBP in a fashion that requires the anti-neuroinflammatory effect of IL-10. While initial thoughts indicated that clinically observed effects of FTY720 were due to lymphoid sequestration [39], new evidence suggests that an effect on glia and specifically astrocytes may underlie FTY720-mediated neuroprotection [13]. S1P regulates cell status and neuroinflammation via S1PR1, S1PR3 and S1PR5 on astrocytes [12; 59], oligodendrocytes and microglia [40]. Astrocytic S1PR1 expression is increased following inflammatory activation by LPS or TNF in vitro [27]. Because astrocytes are a key source of IL-10 in the CNS [32], it is possible that targeting S1PR1 produces an anti-inflammatory shift in astrocytic phenotype in CIBP. Astrocyte-derived IL-10 plays a vital role in regulating microglial phenotype and neurotransmission in the hippocampus [2] and furthermore, astrocyte-derived IL-10 is reported to limit signaling-related neurotoxicity in vivo [61]. Therefore, it is possible that FTY720-mediated antinociception continuously elicits the production of glial IL-10 as a neuromodulator that reduces microglial activation and, critically, limits the extent of activation in the lumbar spinal cord ipsilateral to injury. In conclusion, our work pioneers the characterization of sphingolipid alterations in the lumbar spinal cord 
ipsilateral to painful bone tumor burden using the immune-competent, murine model of metastatic bone disease. We demonstrate that the S1PR1 axis plays a critical role in CIBP, and attenuation of this axis through an IL-10 dependent mechanism abrogates clinically relevant pain behaviors in mice. These findings support the potential fast-track clinical application of the FDA-approved drug, FTY720, as a therapeutic avenue for CIBP.

Acknowledgements. We thank Sayers, N.M. and Symons-Liguori, A.M. for their help with some of the experiments. The authors claim no conflicts of interest. This study was funded by grants from Leukemia and Lymphoma Society 6241-13 (D.S), with additional support from the Saint Louis Cancer Center (D.S.), and by NIH/NCI grants RO1CA142115 (T.W.V.) and NIH/NIGMS R01GM043880 (S.S.). We gratefully acknowledge the VCU Lipidomics and Microscopy Cores, which are supported in part by funding from the NIH-NCI Cancer Center Support Grant P30 CA016059.

\section{Author Contributions.}

SG, HZ, ZC, LS performed experiments and analyzed data; TD analyzed data and assisted in writing manuscript; SS provided sphingolipidomics analysis; SS, TL-M, TV, and DS conceived experiment and wrote manuscript. 


\section{References}

[1] Biology of the Mouse. In: LVM Association editor, Vol. 2016: Louisiana Veterinary Medical Association, 2015.

[2] Almolda B, de Labra C, Barrera I, Gruart A, Delgado-Garcia JM, Villacampa N, Vilella A, Hofer MJ, Hidalgo J, Campbell IL, Gonzalez B, Castellano B. Alterations in microglial phenotype and hippocampal neuronal function in transgenic mice with astrocyte-targeted production of interleukin-10. Brain Behav Immun 2015;45:80-97.

[3] Bigaud M, Guerini D, Billich A, Bassilana F, Brinkmann V. Second generation S1P pathway modulators: research strategies and clinical developments. Biochim Biophys Acta 2014;1841(5):745-758.

[4] Bloom AP, Jimenez-Andrade JM, Taylor RN, Castaneda-Corral G, Kaczmarska MJ, Freeman KT, Coughlin KA, Ghilardi JR, Kuskowski MA, Mantyh PW. Breast cancer-induced bone remodeling, skeletal pain, and sprouting of sensory nerve fibers. The journal of pain : official journal of the American Pain Society 2011;12(6):698-711.

[5] Bolli MH, Abele S, Binkert C, Bravo R, Buchmann S, Bur D, Gatfield J, Hess P, Kohl C, Mangold C, Mathys B, Menyhart K, Muller C, Nayler O, Scherz M, Schmidt G, Sippel V, Steiner B, Strasser D, Treiber A, Weller T. 2imino-thiazolidin-4-one derivatives as potent, orally active S1P1 receptor agonists. Journal of medicinal chemistry 2010;53(10):4198-4211.

[6] Brage ME, Simon MA. Evaluation, prognosis, and medical treatment considerations of metastatic bone tumors. Orthopedics 1992;15(5):589-596.

[7] Brinkmann V, Billich A, Baumruker T, Heining P, Schmouder R, Francis G, Aradhye S, Burtin P. Fingolimod (FTY720): discovery and development of an oral drug to treat multiple sclerosis. Nature reviews 2010;9(11):883-897.

[8] Brinkmann V, Davis MD, Heise CE, Albert R, Cottens S, Hof R, Bruns C, Prieschl E, Baumruker T, Hiestand P, Foster CA, Zollinger M, Lynch KR. The immune modulator FTY720 targets sphingosine 1-phosphate receptors. The Journal of biological chemistry 2002;277(24):21453-21457.

[9] Cai L, Oyeniran C, Biswas DD, Allegood J, Milstien S, Kordula T, Maceyka M, Spiegel S. ORMDL proteins regulate ceramide levels during sterile inflammation. J Lipid Res 2016;57(8):1412-1422.

[10] Caraceni A, Portenoy RK. An international survey of cancer pain characteristics and syndromes. IASP Task Force on Cancer Pain. International Association for the Study of Pain. Pain 1999;82(3):263-274.

[11] Chi XX, Nicol GD. The sphingosine 1-phosphate receptor, S1PR(1), plays a prominent but not exclusive role in enhancing the excitability of sensory neurons. Journal of neurophysiology 2010;104(5):2741-2748.

[12] Choi JW, Gardell SE, Herr DR, Rivera R, Lee CW, Noguchi K, Teo ST, Yung YC, Lu M, Kennedy G, Chun J. FTY720 (fingolimod) efficacy in an animal model of multiple sclerosis requires astrocyte sphingosine 1-phosphate receptor 1 (S1P1) modulation. Proceedings of the National Academy of Sciences of the United States of America 2011;108(2):751-756.

[13] Chun J, Hartung HP. Mechanism of action of oral fingolimod (FTY720) in multiple sclerosis. Clin Neuropharmacol 2010;33(2):91-101.

[14] Cuzzocrea S, Deigner HP, Genovese T, Mazzon E, Esposito E, Crisafulli C, Di Paola R, Bramanti P, Matuschak G, Salvemini D. Inhibition of ceramide biosynthesis ameliorates pathological consequences of spinal cord injury. Shock (Augusta, Ga 2009;31(6):634-644.

[15] Cuzzocrea S, Di Paola R, Genovese T, Mazzon E, Esposito E, Crisafulli C, Bramanti P, Salvemini D. Antiinflammatory and anti-apoptotic effects of fumonisin B1, an inhibitor of ceramide synthase, in a rodent model of splanchnic ischemia and reperfusion injury. J Pharmacol Exp Ther 2008;327(1):45-57.

[16] Doyle T, Finley A, Chen Z, Salvemini D. Role for peroxynitrite in sphingosine-1-phosphate-induced hyperalgesia in rats. Pain 2011;152(3):643-648.

[17] Falk S, Dickenson AH. Pain and nociception: mechanisms of cancer-induced bone pain. J Clin Oncol 2014;32(16):1647-1654.

[18] Finley A, Chen Z, Esposito E, Cuzzocrea S, Sabbadini R, Salvemini D. Sphingosine 1-phosphate mediates hyperalgesia via a neutrophil-dependent mechanism. PloS one 2013;8(1):e55255.

[19] Fujii Y, Hirayama T, Ohtake H, Ono N, Inoue T, Sakurai T, Takayama T, Matsumoto K, Tsukahara N, Hidano S, Harima N, Nakazawa K, Igarashi Y, Goitsuka R. Amelioration of collagen-induced arthritis by a novel S1P1 antagonist with immunomodulatory activities. Journal of immunology 2012;188(1):206-215.

[20] Gatfield J, Monnier L, Nayler O. Ponesimod is a potent S1P1 receptor modulator causing efficient receptor internalisation, degradation and functional antagonism. 28th Congress of the European Committee for Treatment and Research in Multiple Sclerosis. Lyon, France, 2012. p. 926. 
[21] Grisold W, Cavaletti G, Windebank AJ. Peripheral neuropathies from chemotherapeutics and targeted agents: diagnosis, treatment, and prevention. Neuro-oncology 2012;14 Suppl 4:iv45-54.

[22] Hait NC, Avni D, Yamada A, Nagahashi M, Aoyagi T, Aoki H, Dumur CI, Zelenko Z, Gallagher EJ, Leroith D, Milstien S, Takabe K, Spiegel S. The phosphorylated prodrug FTY720 is a histone deacetylase inhibitor that reactivates ERalpha expression and enhances hormonal therapy for breast cancer. Oncogenesis 2015;4:e156.

[23] Hang LH, Li SN, Luo H, Shu WW, Mao ZM, Chen YF, Shi LL, Shao DH. Connexin 43 Mediates CXCL12 Production from Spinal Dorsal Horn to Maintain Bone Cancer Pain in Rats. Neurochem Res 2016;41(5):1200-1208.

[24] Harrison K. Fingolimod for multiple sclerosis: a review for the specialist nurse. Br J Nurs 2014;23(11):582589.

[25] Janes K, Esposito E, Doyle T, Cuzzocrea S, Tosh DK, Jacobson KA, Salvemini D. A3 adenosine receptor agonist prevents the development of paclitaxel-induced neuropathic pain by modulating spinal glial-restricted redox-dependent signaling pathways. Pain 2014;155(12):2560-2567.

[26] Janes K, Little JW, Li C, Bryant L, Chen C, Chen Z, Kamocki K, Doyle T, Snider A, Esposito E, Cuzzocrea S, Bieberich E, Obeid L, Petrache I, Nicol G, Neumann WL, Salvemini D. The development and maintenance of paclitaxel-induced neuropathic pain require activation of the sphingosine 1-phosphate receptor subtype 1. The Journal of biological chemistry 2014;289(30):21082-21097.

[27] Janssen S, Schlegel C, Gudi V, Prajeeth CK, Skripuletz T, Trebst C, Stangel M. Effect of FTY720-phosphate on the expression of inflammation-associated molecules in astrocytes in vitro. Molecular medicine reports 2015;12(4):6171-6177.

[28] Jimenez-Andrade JM, Ghilardi JR, Castaneda-Corral G, Kuskowski MA, Mantyh PW. Preventive or late administration of anti-NGF therapy attenuates tumor-induced nerve sprouting, neuroma formation, and cancer pain. Pain 2011;152(11):2564-2574.

[29] Johnson CH, Patti GJ, Courade JP, Shriver LP, Hoang LT, Manchester M, Siuzdak G. Alterations in Spinal Cord Metabolism during Treatment of Neuropathic Pain. J Neuroimmune Pharmacol 2015;10(3):396-401.

[30] Kays JS, Li C, Nicol GD. Expression of sphingosine 1-phosphate receptors in the rat dorsal root ganglia and defined single isolated sensory neurons. Physiological genomics 2012;44(18):889-901.

[31] Kitatani K, Idkowiak-Baldys J, Hannun YA. The sphingolipid salvage pathway in ceramide metabolism and signaling. Cellular signalling 2008;20(6):1010-1018.

[32] Ledeboer A, Breve JJ, Wierinckx A, van der Jagt S, Bristow AF, Leysen JE, Tilders FJ, Van Dam AM. Expression and regulation of interleukin-10 and interleukin-10 receptor in rat astroglial and microglial cells. The European journal of neuroscience 2002;16(7):1175-1185.

[33] Lee M, Silverman SM, Hansen H, Patel VB, Manchikanti L. A comprehensive review of opioid-induced hyperalgesia. Pain physician 2011;14(2):145-161.

[34] Loram LC, Harrison JA, Sloane EM, Hutchinson MR, Sholar P, Taylor FR, Berkelhammer D, Coats BD, Poole S, Milligan ED, Maier SF, Rieger J, Watkins LR. Enduring reversal of neuropathic pain by a single intrathecal injection of adenosine $2 \mathrm{~A}$ receptor agonists: a novel therapy for neuropathic pain. The Journal of neuroscience : the official journal of the Society for Neuroscience 2009;29(44):14015-14025.

[35] Lozano-Ondoua AN, Hanlon KE, Symons-Liguori AM, Largent-Milnes TM, Havelin JJ, Ferland HL, 3rd, Chandramouli A, Owusu-Ankomah M, Nikolich-Zugich T, Bloom AP, Jimenez-Andrade JM, King T, Porreca F, Nelson MA, Mantyh PW, Vanderah TW. Disease modification of breast cancer-induced bone remodeling by cannabinoid 2 receptor agonists. Journal of bone and mineral research : the official journal of the American Society for Bone and Mineral Research 2013;28(1):92-107.

[36] Lozano-Ondoua AN, Symons-Liguori AM, Vanderah TW. Cancer-induced bone pain: Mechanisms and models. Neuroscience letters 2013;557 Pt A:52-59.

[37] Maiti A, Takabe K, Hait NC. Metastatic triple-negative breast cancer is dependent on SphKs/S1P signaling for growth and survival. Cellular signalling 2017;32:85-92.

[38] Mantyh WG, Jimenez-Andrade JM, Stake JI, Bloom AP, Kaczmarska MJ, Taylor RN, Freeman KT, Ghilardi JR, Kuskowski MA, Mantyh PW. Blockade of nerve sprouting and neuroma formation markedly attenuates the development of late stage cancer pain. Neuroscience 2010;171(2):588-598.

[39] Matloubian M, Lo CG, Cinamon G, Lesneski MJ, Xu Y, Brinkmann V, Allende ML, Proia RL, Cyster JG. Lymphocyte egress from thymus and peripheral lymphoid organs is dependent on S1P receptor 1 . Nature 2004;427(6972):355-360. 
[40] Miron VE, Ludwin SK, Darlington PJ, Jarjour AA, Soliven B, Kennedy TE, Antel JP. Fingolimod (FTY720) enhances remyelination following demyelination of organotypic cerebellar slices. The American journal of pathology 2010;176(6):2682-2694.

[41] Mogil JS, Wilson SG, Bon K, Lee SE, Chung K, Raber P, Pieper JO, Hain HS, Belknap JK, Hubert L, Elmer GI, Chung JM, Devor M. Heritability of nociception I: responses of 11 inbred mouse strains on 12 measures of nociception. Pain 1999;80(1-2):67-82.

[42] Mundy GR. Pathophysiology of cancer-associated hypercalcemia. Seminars in oncology 1990;17(2 Suppl 5):10-15.

[43] Mundy GR. Metastasis to bone: causes, consequences and therapeutic opportunities. Nat Rev Cancer 2002;2(8):584-593.

[44] Nakajima M, Nagahashi M, Rashid OM, Takabe K, Wakai T. The role of sphingosine-1-phosphate in the tumor microenvironment and its clinical implications. Tumour Biol 2017;39(4):1010428317699133.

[45] Nakamura N, Takahashi O, Zenda S, Kawamori J, Ogita M, Onozawa M, Arahira S, Toshima M, Motegi A, Hirano Y, Hojo H, Akimoto T. Neuropathic Pain Features in Patients with Bone Metastases. Clin Oncol (R Coll Radiol) 2016;28(3):204-208.

[46] Nicol GD. Nerve growth factor, sphingomyelins, and sensitization in sensory neurons. Sheng Li Xue Bao 2008;60(5):603-604.

[47] Patti GJ, Yanes O, Shriver LP, Courade JP, Tautenhahn R, Manchester M, Siuzdak G. Metabolomics implicates altered sphingolipids in chronic pain of neuropathic origin. Nature chemical biology 2012.

[48] Portenoy RK, Lesage P. Management of cancer pain. Lancet 1999;353(9165):1695-1700.

[49] Pyne NJ, Pyne S. Sphingosine 1-phosphate and cancer. Nat Rev Cancer 2010;10(7):489-503.

[50] Sabino MA, Mantyh PW. Pathophysiology of bone cancer pain. The journal of supportive oncology 2005;3(1):15-24.

[51] Salvemini D, Doyle T, Kress M, Nicol G. Therapeutic targeting of the ceramide-to-sphingosine 1-phosphate pathway in pain. Trends in pharmacological sciences 2013;34(2):110-118.

[52] Sanchez T, Estrada-Hernandez T, Paik JH, Wu MT, Venkataraman K, Brinkmann V, Claffey K, Hla T. Phosphorylation and action of the immunomodulator FTY720 inhibits vascular endothelial cell growth factor-induced vascular permeability. The Journal of biological chemistry 2003;278(47):47281-47290.

[53] Sloane E, Langer S, Jekich B, Mahoney J, Hughes T, Frank M, Seibert W, Huberty G, Coats B, Harrison J, Klinman D, Poole S, Maier S, Johnson K, Chavez R, Watkins LR, Leinwand L, Milligan E. Immunological priming potentiates non-viral anti-inflammatory gene therapy treatment of neuropathic pain. Gene therapy 2009;16(10):1210-1222.

[54] Spiegel S, Milstien S. The outs and the ins of sphingosine-1-phosphate in immunity. Nature reviews Immunology 2011;11(6):403-415.

[55] van den Beuken-van Everdingen MH, de Rijke JM, Kessels AG, Schouten HC, van Kleef M, Patijn J. Prevalence of pain in patients with cancer: a systematic review of the past 40 years. Ann Oncol 2007;18(9):1437-1449.

[56] Verzijl D, Peters SL, Alewijnse AE. Sphingosine-1-phosphate receptors: zooming in on ligand-induced intracellular trafficking and its functional implications. Mol Cells 2010;29(2):99-104.

[57] Welch SP, Sim-Selley LJ, Selley DE. Sphingosine-1-phosphate receptors as emerging targets for treatment of pain. Biochemical pharmacology 2012;84(12):1551-1562.

[58] WHO. Cancer pain relief. With a guide to opioid availability. Geneva: World Health Organization, 1996.

[59] Wu C, Leong SY, Moore CS, Cui QL, Gris P, Bernier LP, Johnson TA, Seguela P, Kennedy TE, Bar-Or A, Antel JP. Dual effects of daily FTY720 on human astrocytes in vitro: relevance for neuroinflammation. J Neuroinflammation 2013;10:41.

[60] Yang Y, Zhang J, Liu Y, Zheng Y, Bo J, Zhou X, Wang J, Ma Z. Role of nitric oxide synthase in the development of bone cancer pain and effect of L-NMMA. Molecular medicine reports 2016;13(2):1220-1226.

[61] Zhou Z, Peng X, Insolera R, Fink DJ, Mata M. Interleukin-10 provides direct trophic support to neurons. Journal of neurochemistry 2009;110(5):1617-1627.

[62] Zhu S, Wang C, Han Y, Song C, Hu X, Liu Y. Sigma-1 Receptor Antagonist BD1047 Reduces Mechanical Allodynia in a Rat Model of Bone Cancer Pain through the Inhibition of Spinal NR1 Phosphorylation and Microglia Activation. Mediators of inflammation 2015;2015:265056. 


\section{Figure captions.}

Figure 1. Decreased ceramide and increased S1P in spinal cords of 66.1 bone tumor bearing mice, a model of cancer-induced bone pain. Lumbar spinal cord ipsilateral to the site of arthrotomy was harvested 14 days postsurgery from sham and 66.1 tumor-bearing mice and sphingolipid levels determined by LC-ESI-MS/MS. (A) Total ceramide and ceramide acyl chain species. (B) Total monohexosylceramide and its acyl chain species. (C) Total sphingomyelin and its acyl chain species. (D) Sphingosine and (E) S1P. Graphs are representative of two individual experiments and lipidomic analyses. Data are expressed as mean \pm SEM for $n=5$ mice/group and analyzed by Welch's corrected, unpaired, one-tailed Student's t-test. False discovery rate was controlled by Benjamini-Hochberg procedure $\left(\mathrm{q}<0.05 ; \mathrm{q}^{*}=0.031\right)$. $* \mathrm{P}<0.03$ vs. sham.

Figure 2. Enhanced de novo sphingolipid biosynthesis and increased dihydro-S1P levels in spinal cords of 66.1 bone tumor bearing mice. Lumbar spinal cord ipsilateral to the site of arthrotomy was harvested 14 days postsurgery from sham and 66.1 tumor-bearing mice and dihydrosphingolipid levels determined by LC-ESIMS/MS. (A) Total dihydroceramide and its acyl chain species. (B) Total monohexosyldihydroceramide and its acyl chain species. (C) Total dihydrosphingomyelin and its acyl chain species. (D) Dihydrophingosine and (E) dihydro-S1P. Data are representative of two individual experiments and expressed as mean \pm SEM for $n=5$ mice/group and analyzed by Welch's corrected, unpaired, one-tailed Student's t-test. False discovery rate was controlled by Benjamini-Hochberg procedure $\left(\mathrm{q}<0.05 ; \mathrm{q}^{*}=0.031\right) . * \mathrm{P}<0.03$ vs. sham.

Figure 3. S1PR1 antagonists attenuate spontaneous flinching and guarding CIBP behaviors. Mice were evaluated on day 11 when they displayed $\geq 5$ flinches and $\geq 10$ seconds guarding over a 2-minute period. Treatment was administered at $t=0$ and mice were tested until the effect resolved. (A) When compared to vehicle (3\% DMSO control; $\diamond ; n=6$ ), intrathecal administration of TASP0277308 (3 nmol; $\mathbf{\square} ; \mathrm{n}=9$ or $10 \mathrm{nmol}$; $\boldsymbol{\Delta} ; \mathrm{n}=8)$ or FTY720 (3 nmol; $\mathbf{O} ; \mathrm{n}=7$ ) attenuated CIBP-induced flinching. (B) Likewise, guarding behaviors were attenuated with intrathecal administration of TASP0277308 (3 nmol; $\mathbf{a} ; \mathrm{n}=9$ or $10 \mathrm{nmol} ; \boldsymbol{\Lambda} ; \mathrm{n}=8)$ or FTY720 (3 nmol; $\bigcirc$; $=7$ ) when compared to vehicle ( $\diamond ; n=4)$. Dose-response flinching (C) and guarding (D) behaviors to intraperitoneal FTY720 (0.1, $;$; 0.3, $\mathbf{\square}$; or $1.0 \mathrm{mg} / \mathrm{kg}, \boldsymbol{\Delta} ; \mathrm{n}=8)$ compared to vehicle $(\diamond ; \mathrm{n}=4)$. Data are expressed as mean \pm SEM for (n) animals and analyzed by two-tailed two-way ANOVA with Bonferroni comparisons. $* \mathrm{P}<0.05$ vs. $\mathrm{t}=0 \mathrm{~h}$.

Figure 4. Systemic administration of FTY720 is not subject to tolerance. FTY720 (1 mg/kg/d, i.p. $)$ or vehicle (5\% DMSO, i.p.) was administered once daily from post-surgical days 7-14. Flinching (A) and guarding (B) behaviors are shown before and $1 \mathrm{~h}$ after drug administration on the first (day 7) and last (day 14) days of 
treatment. Data are expressed as mean \pm SEM for $n=10$ mice/group and analyzed by two-tailed two-way ANOVA with Bonferroni comparisons. $* \mathrm{P}<0.05$ vs. sham and $\dagger \mathrm{P}<0.05$ vs. daily baseline.

Figure 5. The beneficial effects of FTY720 on CIBP are IL-10-dependent. On post-surgical day 7 when mice exhibited sufficient CIBP behaviors ( $>5$ flinches and $>10$ seconds guarding over a 2-minute period, days 1014), mice were implanted with 7-day subcutaneous minipumps containing FTY720 (1 mg/kg/d). When measured on days 10 and 14, FTY720 micropump infusion reversed CIBP flinching (A) and guarding (B) behaviors. The ability of FTY720 to maintain reversal of CIBP behaviors was attenuated $1 \mathrm{~h}$ after intrathecal administration of IL-10 neutralizing antibody ( $\alpha$ IL10; black arrow; $\mathrm{n}=8)$, but not IgG control $(\mathrm{n}=5)$. Data are expressed as mean \pm SEM for (n) mice and analyzed by two-tailed, two-way ANOVA with Bonferroni comparisons. \#P<0.05 vs. D10 or D14.

Figure 6. Repeated administration of FTY720 does not alter bone metabolism. FTY720 (1 mg/kg/d, i.p.) or vehicle (5\% DMSO, i.p.) was administered once daily from post-surgical days 7-14. (A) Sample post-surgical day 14 sham and tumor-bearing radiographs showing radiotranslucent lesions of bone loss (white arrows). (B) Bone loss ratings obtained from blinded evaluation of radiographs on post-surgical days 0 , 7, and 14 ( $n=10 /$ group). (C) C-telopeptide fragment of collagen type I (CTX) was measured in serum harvested from animals on post-surgical day 14 ( $n=5$ /group). Data are expressed as mean \pm SEM for (n) mice per group and analyzed by two-tailed two-way (B) or one-way (C) ANOVA with Bonferroni comparisons. $* P<0.05$ vs. sham. 
Fig. 1

Sphingolipid degradation

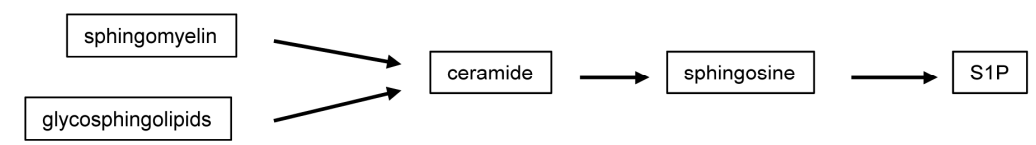

A

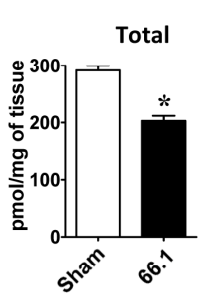

Ceramides

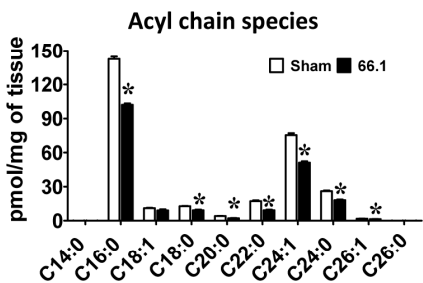

C

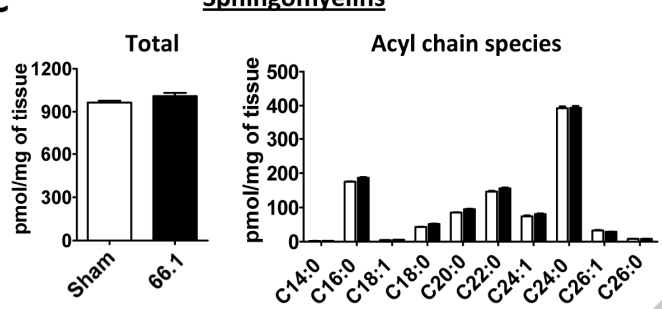

B

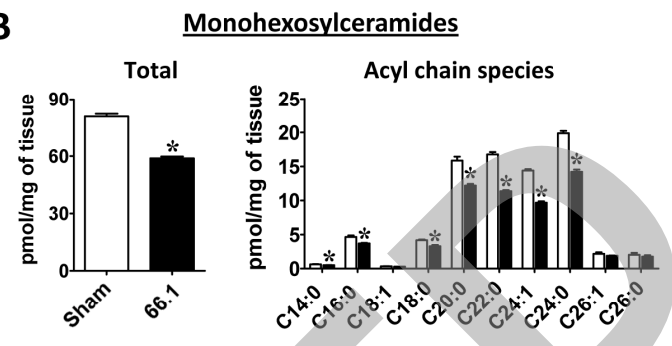

D

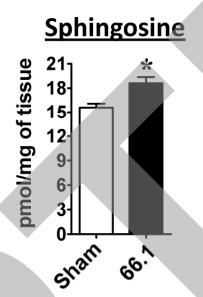

E

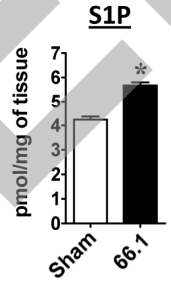


Fig. 2

de novo sphingolipid biosynthesis

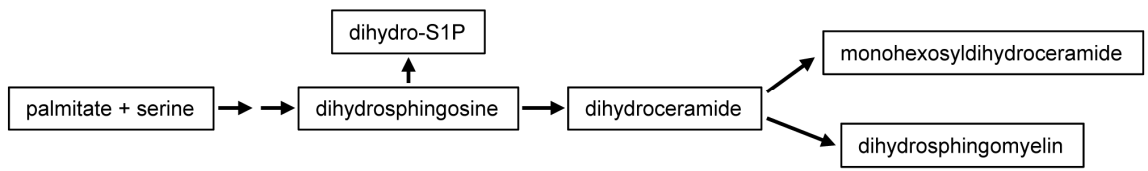

A

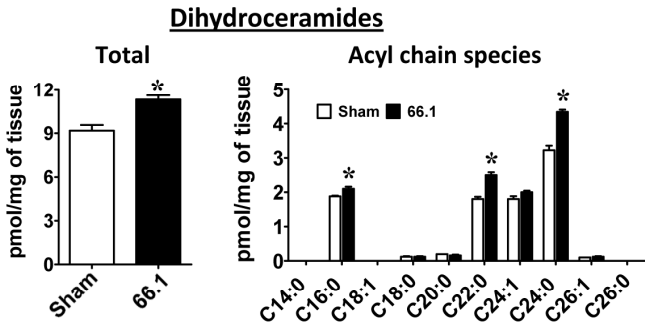

C

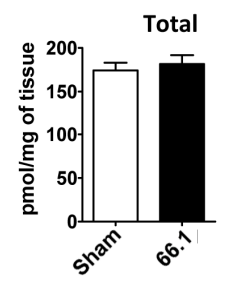

Dihydrosphingomyelins

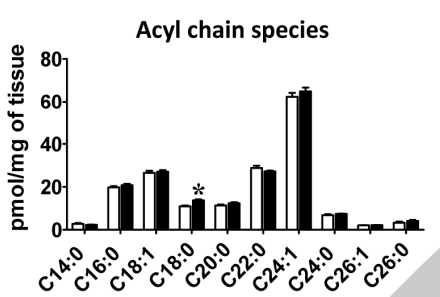

B Monohexosyldihydroceramides

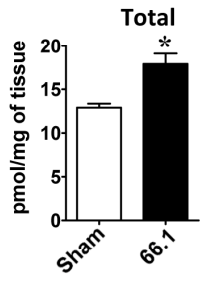

Acyl chain species

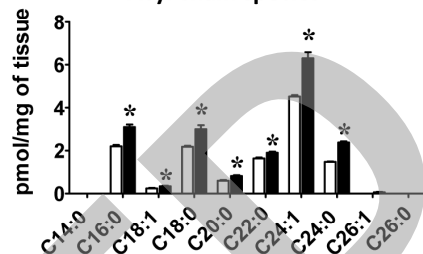

D

Dihydrosphingosine

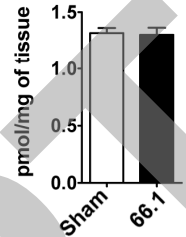

E Dihydro-S1P

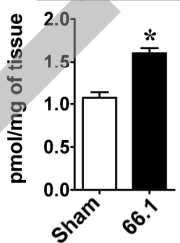


Fig. 3 Flinching Behavior

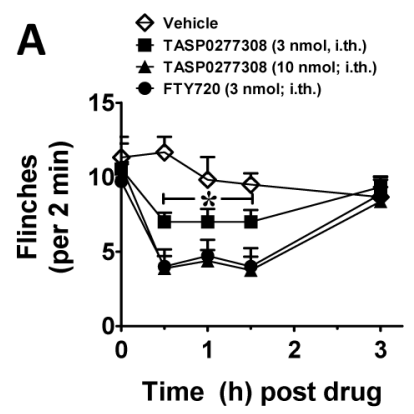

C $\begin{aligned} & \diamond \text { Vehicle } \quad 0.3 \mathrm{mg} / \mathrm{kg} \\ & -0.1 \mathrm{mg} / \mathrm{kg} \quad \neq 1 \mathrm{mg} / \mathrm{kg}\end{aligned}$

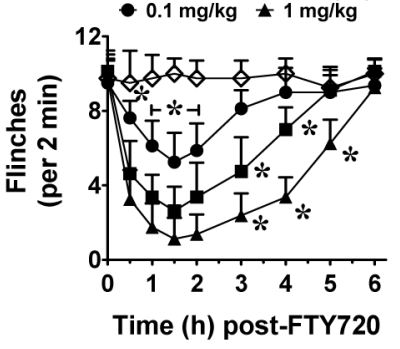

Guarding Behavior
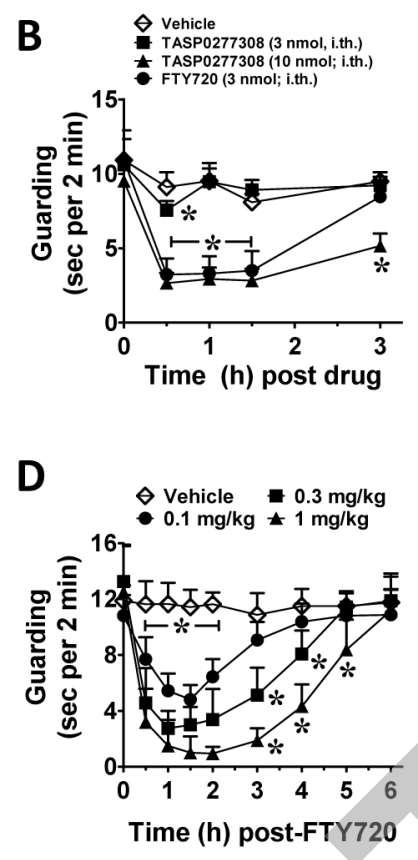
Fig. 4
$\square$ Sham + Vehicle
Sham + FTY720 (1 mg/kg/d, i.p.)
$66.1+$ Vehicle
$66.1+$ FTY720 (1 mg/kg/d, i.p.)

A

Flinching Behavior

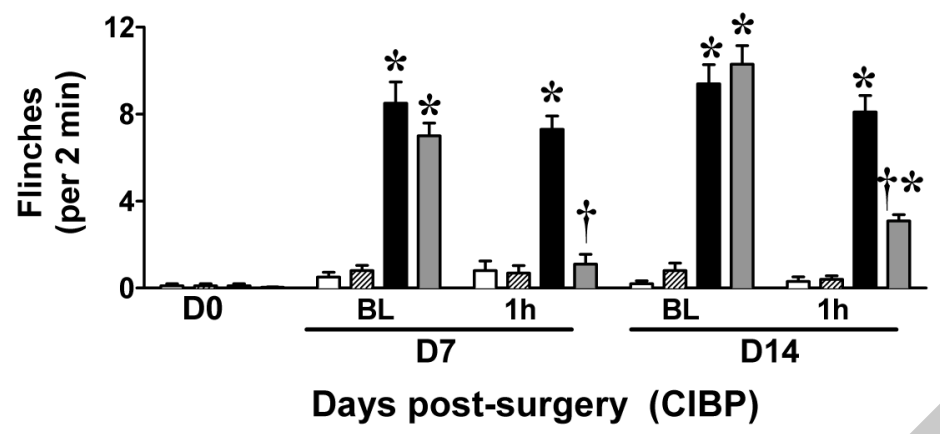

B
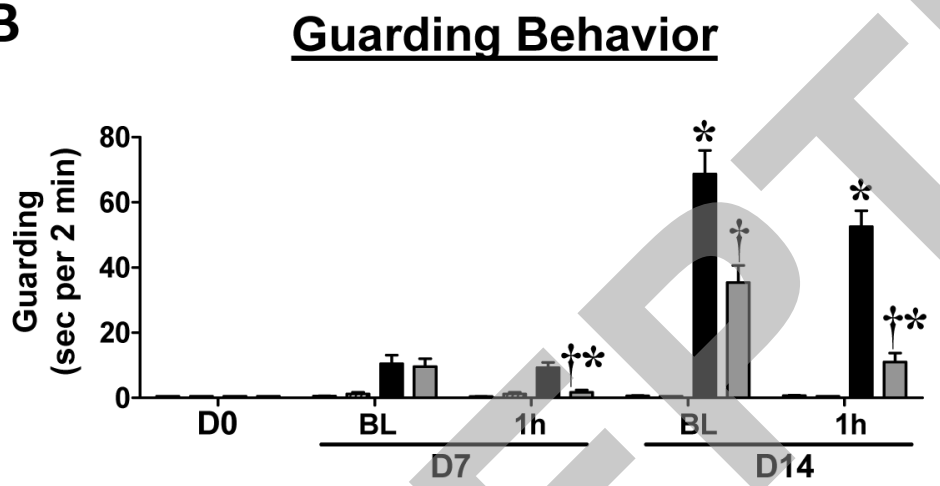

Days post-surgery (CIBP) 
<smiles>C=CC</smiles> 
Fig. 6

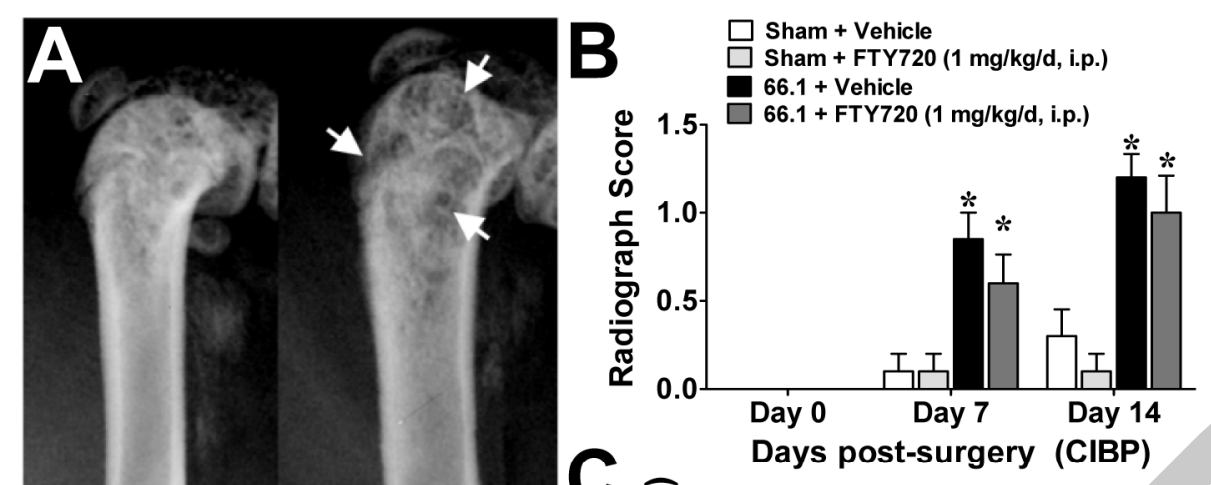

Sham

66.1

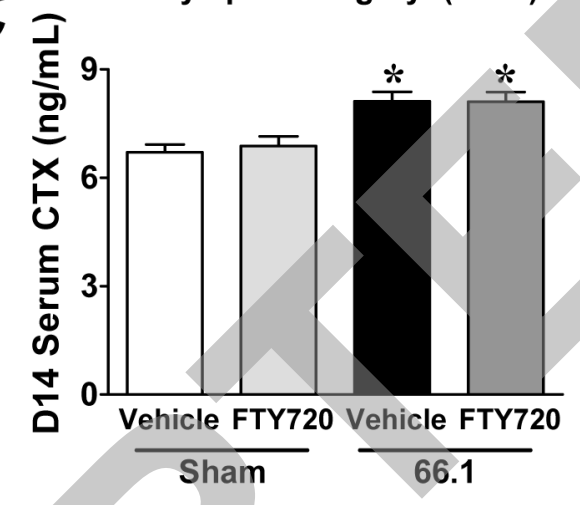

